\title{
LA EXPRESIÓN CORPORAL Y EL DESARROLLO MOTOR DE NIÑOS DE 3 AÑOS
}

\section{BODY EXPRESSION IN GROSS MOTOR DEVELOPMENT IN 3-YEAR-OLDS.}

\author{
Mario Paúl Simbaña Haro ${ }^{1}$ \\ Mario Gerardo Gonzalez Romero 2 \\ Cristina Elizabeth Merino Toapanta ${ }^{3}$ \\ Dayana Estefanía Sanmartin Lazo ${ }^{4}$
}

Recibido: 2021-10-08 / Revisado: 2021-11-05 / Aceptado: 2021-11-25 / Publicado: 2022-01-01

\begin{abstract}
Forma sugerida de citar: Simbaña-Haro, M. P., Gonzalez-Romero, M. G., Merino-Toapanta, C. E., SanmartinLazo, D. E. (2022). Expresión corporal en el desarrollo motor del niño de 3 años. Retos de la Ciencia. 6(12). 2540. https://doi.org/10.53877/rc.6.12.20220101.03
\end{abstract}

\section{RESUMEN}

La expresión corporal es una manera de comunicación del ser humano, va acompañada de gestos, expresiones faciales y posturas, los cuales, representan sentimientos, emociones, pensamientos e ideas. Todas estas manifestaciones expresan permanentemente y van asociadas del desarrollo motor grueso, en el cual, domina la armonía y el control del movimiento, logrando una adecuada sincronización y coordinación motora. Desde esta perspectiva, el estudio tubo como objetivo describir la relacion de la expresión corporal en el desarrollo motor grueso de niños de 3 años. Al ser una investigación de enfoque cualitativo, la información se recogió a través de la aplicación de una guiá de observación con destrezas relacionadas a la expresión corporal y a la motricidad gruesa, dirigida a 32 niños de 3 años. Obteniéndose como resultado, que un buen porcentaje de niños se encuentra iniciando su desarrollo en el manejo de movimientos articulatorios básicos, e imitación de movimientos en canciones, seguir ritmos, así como la identificación de emociones y sentimientos de las personas de su entorno; y en lo referido a las destrezas de motricidad gruesa como el de realizar ejercicios de equilibrio dinámico y estático con

1 Magíster en Gestión Educativa y Desarrollo Social. Docente Investigador de la Universidad Tecnológica Indoamérica. Ecuador. E-mail: mariosimbana@indoamerica.edu.ec / ORCID: https://orcid.org/0000-0001-82093391

2 Magíster en Administración Educativa y Docencia Universitaria. Docente Investigador de la Universidad Tecnológica Indoamérica. Ecuador. E-mail: mariogonzalez@indoamerica.edu.ec / ORCID: https://orcid.org/00000003-2292-3220

3 Magister en Educación Inicial. Docente de la Universidad Tecnológica Indoamérica. Ecuador. E-mail: mcristina@indoamerica.edu.ec / ORCID: https://orcid.org/0000-0002-9275-0727

4 Licencienciada en Ciencias de la Educación Inicial. Estudiante de la Universidad Tecnológica Indoamérica. Email: d.sanmartin@uti.edu.ec / ORCID: https://orcid.org/0000-0001-9313-9594 
las diferentes formas de movimientos, caminar, correr y mantener el control postural que involucre movimientos segmentados de su cuerpo, cabeza, tronco y extremidades; también, en un buen porcentaje de niños se encuentran en inicado su desarrollo. Lo que lleva a asumir que la expresión corporal se encuentra íntimamente relacionado con el desarrollo de habilidades motoras gruesas y que estas ayudan a que los niños desde tempranas edades a que logren una mejor comunicación y expresión.

Palabras clave: expresión corporal, motricidad gruesa, dominio corporal dinámico, dominio corporal estático.

\section{ABSTRACT}

Body expression is a way of communication typical of each human being, it is accompanied by gestures, facial expressions, postures which represent feelings, emotions, thoughts and ideas. All these manifestations are permanently reflected and are associated with gross motor development, in which harmony and movement control dominates, achieving adequate motor synchronization and coordination. From this perspective, the study aimed to describe the relationship of body expression in gross motor development of 3-year-old children. Being a qualitative approach research, the information was collected through the application of an observation guide with skills related to body expression and gross motor skills, aimed at 32 3-yearold children. Obtaining as a result the following, that a good percentage of children is beginning their development in the management of basic articulatory movements, and imitation of movements in songs, following rhythms, as well as the identification of emotions and feelings of the people around them; and with regard to gross motor skills such as performing dynamic and static balance exercises with different forms of movement, walking, running and maintaining postural control that involves segmented movements of your body, head, trunk and extremities, as well, in a similar percentage of children are in the beginning of their development. Which leads us to establish that body expression is closely related to the development of gross motor skills and that these help children from an early age to achieve better communication and expression.

Keywords: body expression, gross motor skills, dynamic body control, static body control.

\section{INTRODUCCIÓN}

El nivel de educación inicial se oficializa y forma parte del sistema educativo cuando se inserta en la Constitución de la República del Ecuador en el año 2008, nivel creado para brindar servicios educativos a niños de 0 a 5 años el mismo que está organizado en dos subniveles, el sub nivel 1 de educación inicial ( 0 a 3 años) y el sub nivel 2 de Educación Inicial (3 y 4 años); en los que se busca, que el niño desarrolle habilidades y destrezas y tengan mayores posibilidades de desarrollo en los niveles superiores. Con este propósito el Ministerio de Educación ha creado el currículo de educación inicial fundamentado en el derecho a la educación de los menores de cinco años. Prioriza el desarrollo cognitivo, afectivo y motriz, lo último es un elemento fundamental que es necesario que el niño los desarrolle, para que pueda expresar sus emociones y sentimientos, mediante su cuerpo. 
El Centro de Desarrollo Infantil Orquídeas se encuentra ubicado al sur de Quito en la ciudadela Ibarra, Barrio las Orquídeas, oferta un servicio que pertenece a la CDI que oferta el MIES, tiende a 63 niños de 0 a 5 años de familias de escasos recursos y vulnerabilidad.

El centro infantil cumple con las normas de regulación, seguridad de acuerdo con los parámetros establecidos por la Secretaría Nacional de Gestión de Riesgos. (MIES, 2014). Las actividades académicas se realizan permanentemente mediante planificaciones didácticas elaboradas para desarrollar las destrezas dadas en el currículo del nivel, bajo ámbitos de aprendizaje, con la metodología juego - trabajo y en rincones de aprendizaje.

Se ha observado que los niños tienen dificultades en el manejo de la expresión corporal en el desarrollo motor grueso como subir y bajar gradas coordinadamente, carreras, caminar, saltos, arrastre, reptar y salticar coordinadamente, esto ocasiona que el niño tenga dificultades para escribir, calcular direccionarse y también para integrarse, socializar, participar, y expresar sus ideas pensamiento e ideas sin temor a la equivocación, generando un déficit de desarrollo cognitivo, afectivo, social y motriz. De lo anterior se formula lo siguiente interrogante de la investigación, ¿cuál es la relación entre la expresión corporal y el desarrollo motor grueso de los niños de 3 años del Centro de Desarrollo Infantil "Orquídeas" en el periodo académico 20192020? Una vez terminada, la investigación busca describir la relacion de la expresión corporal en el desarrollo motor grueso de niños de 3 años, contribuirá al perfil de salida de los niños participantes, se evidencia más actividades orientadas al esquema corporal como herramienta principal en adquisición de nuevos conocimientos y desarrollo del parte motor grueso, para ello el docente es clave en poder lograr lo anteriormente descrito.

En el Plan Nacional para el Buen Vivir en el cual estipula lo siguiente; "el desarrollo integral es una prioridad y política pública", (Consejo Nacional de Planificacion, 2015) esto permite el acceso a una educación de calidad, que potencialice capacidades y no solo se enmarque en la adquisición de conocimiento sino también en las experiencias de vida que aporta el medio social.

Partiendo del desarrollo integral, el Currículo de Educación Inicial se sujeta a una base teórica donde se toma algunas características que aportan al diseño del mismo como integralidad, inclusión, familia; es así que (Vigotstky, 1974) plantea un aprendizaje dividido o zonas de desarrollo los cuales se determinan periodos sensibles ante un conocimiento, nos habla también de las relaciones sociales ya que es propio de los niños aprender mediante la interacción e imitación de sus pares y otras personas de su entorno, por tal motivo es de mucha importancia que los padres, docentes y familiares garanticen un ambiente estimulante en sus primeros años de vida lo que ayudara a que el niño se desenvuelva de una manera oportuna y no tenga temor de expresar lo que siente y piensa.

En cambio, para Rogoff (1993), la educación del niño en sus primeros años es de participación, donde todo lo que el comparte y juega se relaciona le genera un aprendizaje significativo que le permite desarrollarse con rasgos y características únicas.

El arendizaje significativo explicado por Ausubel, plantea que el niño es el creador de su propio conocimiento, sobre una idea general este conocimiento es estructurado en base de los intereses y necesidades logra una valoración propia, esto mejora su capacidad comprensiva para, lo cual, se debe contar con la motivación positiva del niño hacia el nuevo aprendizaje. (Curriculo de Educación Inicial, 2017, p. 14) 
Con los aportes de los autores mencionados se puede definir al currículo como un proceso sistemático de interrelaciones aprendizajes que potencializarán el desarrollo de habilidades y valores a lo largo de su vida, por ello, es importante que mientras aprenden creen experiencias nuevas, exploren a partir de su propio cuerpo, reconozcan ambientes seguros descubran su medio, logren expresase y comunicarse mediante un lenguaje verbal y corporal, logrando una excelente convivencia en el entorno natural y cultural.

Es por ello que, el currículo se centra en los aspectos cognitivos, sociales, psicomotrices, físicos y afectivos de cada niño, garantizando un desarrollo integral, además se considera que para que el niño alcance su bienestar, implica la satisfacción de sus necesidades básica si la familia y la unidad de atención logra dar relevancia a este punto se evidenciara actitudes involuntarias en los niños como es la espontaneidad, felicidad, autoestima, vitalidad, salud, movimiento y demostrara ser un niño con valores que se siente querido y activo e importante.

Un elemento de gran importancia que se viene trabajando en Educación Inicial es el sentir, pensar y actuar los cuales se enmarcan en la organización curricular de los aprendizajes o también llamados ejes de desarrollo y aprendizaje con sus diferentes ámbitos correspondientes así tenemos; eje de descubrimiento natural y cultural que es el proceso cognitivo de niño, es el pensar, desarrollo personal y social, lo que permite que el niño sea capaz de expresar lo que siente sin reprimirse, desenvolviéndose con su propia identidad marcando su personalidad y confianza y por último, el eje de expresión y comunicación, que concentra las diversas manifestaciones que tiene el niño para poder expresar sus ideas y deseos a los demás.

En esta investigación se va a enfocar en el eje de expresión y comunicación para desarrollar diferentes lenguajes que permitan relacionarse positivamente con los demás.

Al hablar del eje de expresión y comunicación, se lleva acabo procesos para desarrollar la capacidad comunicativa y expresiva de los niños empleando diversas manifestaciones, así como es el lenguaje verbal y el no verbal como medios de expresión de sus ideas, pensamientos, actitudes, experiencias y emociones que permiten llevar a cabo relaciones positivas con las personas de su entorno. (Toro Santacruz, Soraya; Espinoza Salas, Maria, 2014, p 20).

El desarrollo pleno de la comunicación y el lenguaje se encuentran ligada al desarrollo de otras habilidades como es la percepción, el pensamiento y las relaciones del entorno, además en niños de edad escolar posibilitaran la inserción favorable a la escritura.

Por otro lado el ámbito de exploración del Cuerpo y Motricidad, contempla el desarrollo de las habilidades motrices básicas, así como; los primeros movimientos del cuerpo como son los reflejos siendo una etapa transitoria y de evolución permanente tanto para el niño como para sus padres, este periodo permite la coordinación de huesos, músculos, tendones y nervios los cuales posibilitan las diferentes acciones del ser humano, el desarrollo motor es el reflejo del sistema nervioso de tal manera permite detectar posibles problemas a edad temprana. (García, 2019)

Cuando el niño ha pasado por el proceso de gateo y comienza su proceso de bipedestación y da sus primeros pasos, aumentan sus posibilidades de interacción y conocimiento de su entorno inmediato, el autoconocimiento de su propio cuerpo permitirá el logro del reconocimiento del esquema corporal. 
El movimiento progresivo determinara acciones futuras como es el correr, saltar, trepar, lanzar y otras actividades que domine su cuerpo además de los movimientos finos, todas estas cualidades definen también el equilibrio, velocidad direccionalidad y coordinación consigo mismo y con el objeto.

El Ministerio de Inclusión Económica y Social en la guía metodológica de los servicios de Centros Infantiles del Buen y Vivir y Centros de Desarrollo Infantil (2015) establece que, "Este ámbito toma en cuenta cuatro grandes aspectos vinculados al desarrollo motor como es la coordinación dinámica global, la relajación, la disociación de movimientos y la eficacia motriz". (p. 15). Estos logros evidenciados en los niños permiten hacer una medición de avances tomando en cuenta los rasgos particulares.

En cambio en el Currículo de Educación inicial para el sub nivel 2 contempla características muy similares aunque mantiene otro nivel de progresividad, en el ámbito Comprensión y expresión del lenguaje, permite exteriorizar sus pensamientos, ideas, sentimientos, reacciones y vivencias de manera más clara mediante la utilización de símbolos verbales y no verbales además de diversos lenguajes y lenguas, es de gran importancia dar sentido a esta edad ya que significara la base para procesos futuros en cuanto a lo que es lectura y escritura.

La expresión corporal y motricidad en el subnivel dos propone mejorar las habilidades motrices que ya se han venido trabajando en el anterior nivel e integrar conocimientos partiendo de sus experiencias de aprendizaje como es el conocimiento de su propio cuerpo sus funciones y su importancia. (Curriculo de Educación Inicial, 2014, p. 32). Sin embargo, para alcanzar todas las destrezas relacionadas a cada eje y ámbito se debe trabajar juntamente con la familia brindando una buena y adecuada estimulación temprana posibilitando al niño mayores alcances

Expresión corporal o también llamado lenguaje corporal es una forma de comunicación en la cual no interviene la parte verbal. Todos los gestos y movimientos que el cuerpo emite es un guía de nuestro pensar y sentir. La expresión corporal es un medio creativo un canal transmisor de emociones, siendo emisor o receptor tienen la capacidad de absorber o recibir por sus sentidos, todas las cargas de energía al momento de relacionarse con las demás personas y de dar respuestas a estas impresiones con una herramienta propia como es el cuerpo. Rodriguez (2008). Menciona que trabajar a muy temprana edad la expresión corporal ayuda a generar sensibilidad al momento de poder intuir conductas, enriquecer la creatividad e imaginación, adquirir seguridad de sí mismo de su cuerpo de sus facciones, genera buena conducta de modo que proyecta un pensamiento abierto, reflexivo y crítico acompañado de un buen humor. Ayuda a valorar su cuerpo de tal modo que incide en el desarrollo motor grueso permitiendo razonar y moverse (p.127).

Para Patricia Stoke creadora de la expresión corporal y danza la expresión corporal es la fuente principal del cuerpo es universal transparente e innato ayuda a que el ser humano se desarrolle de manera integral. Citado por (Blanco, 2009)

Por lo tanto, la expresión corporal se define como un medio de comunicación eficaz natural y espontaneo donde se puede exponer nuestra personalidad permitiendo al cuerpo liberar energías que permitirá interactuar con el medio que lo rodea incrementar su imaginación y desarrollar su creatividad siendo personas libres. En educación inicial la expresión corporal permite que el niño logre trasmitir lo que siente o desea en ese momento refleja también su estado anímico y también se lo considera como medio de información de lo que le puede suceder o padece en casa.

La importancia que tiene la expresión corporal es que el cuerpo lo relacionamos como si fuera una casa en la cual habitamos todos los días y es el primer medio de partida de los aprendizajes ya que mediante de él conocemos nuestro entorno, 
relacionamos vivencias, experimentamos cambios, es un medio que a más de manifestar nuestro pensar permite reconocer habilidades destrezas cualidades y capacidades en diferentes aprendizajes propuestos, tiene la característica de ser creativos e innovadores llenos de imaginación donde se permite la espontaneidad, goce e improvisación. Mantener un lenguaje expresivo es de importancia porque así da a conocer el dominio de la expresión y comunicación a las demás personas, para ello se utiliza diversas artes como es el teatro, danza, mimo no solo como una disciplina sino también como una manera de ayuda emocional y de mantener la mente y el cuerpo estable logrando una estabilidad.

Cada ser humano posee su propio esquema de desarrollo, lo cual define su individualidad. Nace dotado de características que determinan su modo de ser, de actuar y de pensar. Todo lo que hace para conocer, para relacio-narse, para aprender, lo hace a través del cuerpo. Sus primeras experiencias vividas son esencialmente corporales, imprimiendo huellas indelebles en su inconsciente corporal. (Leni y Wey, 2015, p.25)

Otro punto que no debe pasar por desapercibido son los actores de la educación, aspecto de igual importancia en el desarrollo de los niños, la expresión corporal debe ser trabajada como una disciplina que conlleva al desarrollo integral esto va a influir en los aspectos físico, emocional, cognitivo y social, sin embargo en las aulas para algunos docentes el hecho que el niño, no moleste o no hable es sinónimo de niño educado, esto es una equivocación porque el permanecer en silencio o quedarse quieto, es una forma de expresión que algo anda mal, por naturaleza los niños son inquietos y en edades tempranas es vitalidad, estos signos se debe tomar muy en cuenta por parte del educador.

De acuerdo con Boulch (1973), en su obra titulada la psicomotricidad establece que "El esquema corporal es una intuición global o conocimiento inmediato que nosotros tenemos de nuestro propio cuerpo, tanto en estado de reposo como en movimiento, en relación con sus diferentes partes y sobre todo con relación al espacio y con los objetos que lo rodean". (p.39). Comprende que el esquema corporal es el todo del conocimiento de nuestro cuerpo, sea en movimiento o en reposo en relación con un espacio, constituye también la imagen del cuerpo como posturas, lateralidad y movimientos desenvolviéndose en su entorno.

Según Piaget se obtiene al esquema corporal por las nociones cognitivas del cuerpo es decir cuando el niño se enfrenta a relaciones tempero- espaciales, teniendo en cuenta la ubicación del cuerpo en relación de otros objetos o personas así también la permanencia de los objetos, aunque este no esté presente.

El esquema corporal es la idea que poseemos del propio cuerpo, sus partes y movimientos, generando así una idea mental, este proceso se va dando de manera progresiva mediante la experiencia y vivencias consecuencia de la maduración del niño, una vez que este término sea conocido los infantes serán capaces de controlar sus movimientos y la fuerza motriz que utilizan. Existen factores que influyen en la construcción del esquema corporal como la percepción, el movimiento, lo cognitivo y el lenguaje.

Si conseguimos que una persona se permita una expresión-comunicación mássincera. Que se permita expresar adecuadamente sus sentimientos y deseos. Si posi-bilitamos que una persona mejore sus sistemas de escucha, de atender y valorar alotro, recibiendo y entendiendo sus mensajes, no solo verbales, sino también los noverbales provenientes del paralenguaje y del lenguaje no verbal, estaremos mejoran-do los sistemas de comunicación de esa persona. (Montesinos 2017, p. 39) 
Para alcanzar el desarrollo integral del niño es necesario conocer la estructuración del esquema corporal para desarrollar este proceso es necesario que los docentes ayuden a construir su identidad, por lo que se debe utilizar recursos apropiados para trabajar de manera lúdica en espacios de seguridad para el niño, en educación infantil la finalidad es lograr un desarrollo físico, afectivo. Intelectual y social partiendo de su autoconocimiento.

Entre tanto, el lenguaje corporal si bien el cuerpo es utilizado como instrumento de expresión también interviene los gestos y el movimiento esto hace que el mensaje sea más entendible. El lenguaje del cuerpo puede ser independiente frente al lenguaje verbal, este sin número de movimientos que se pueden emitir son estudiados por la kinésica o quinésica. Dentro del lenguaje corporal tenemos algunos aspectos como son:

La postura corporal, es la postura que adopta nuestro cuerpo o sus partes en orientación a otro cuerpo, existen postras abiertas y cerradas, las primeras consisten en no utilizar los brazos como una barrera sutilmente se colocara a la altura de la cadera, y la cerrada es cuando los brazos se encuentran cruzados, son posturas inconscientes que refleja seguridad o inseguridad. Así también la postura de la cabeza refleja algunas situaciones perceptibles como son los movimientos de un lado hacia el otro significa negación, de arriba hacia abajo, asentimiento, aseguración correspondencia, inclinada interés e inclinada hacia abajo desaprobación, negación, no es una buena actitud

Los gestos expresan estados emocionales o muestras de afecto, por ejemplo, ansiedad, alegría, nerviosismo o tención, en cambio los gestos de adaptación son aquellos que ayudan a relajarnos o tranquilizarnos por ejemplo tomar un lápiz en nuestras manos cuando expresamos algo otros gestos también pueden ser inconscientes como morderse las uñas o chuparse los dedos esto pasa a menudo en los niños pequeños.

Según Balseca (2013), en su guía docente de la expresión corporal afirma que: El lenguaje corporal posee una gran ventaja, al momento de contar con nuestro cuerpo nuestros sentimientos, por ello se relaciona también la frase "una acción vale más que mil palabras". El cuerpo es demasiado inteligente a carencia de alguna capacidad trata de acoplarse así pasa con los niños y adultos que poseen alguna discapacidad, por ejemplo: los sordomudos perdieron la capacidad auditiva y vocal ante su dificultad ha logrado desarrollar el lenguaje de señas que es un lenguaje corporal. (Balsec, 2013, p.19)

Entre tanto, la memoria corporal conserva la información del cuerpo y lo que este percibe, gracias al contacto corporal el niño logra imprimirse y verse como una figura estructurada, en los bebes por ejemplo necesita que su madre o toque y le hable esta estimulación ayudara a tener conciencia de su cuerpo, mediante nuestra piel se logra captar diferentes texturas, temperaturas, etc.

La psicomotricidad es parte fundamental del desarrollo integral de la persona, es el movimiento esencial que nos da a conocer el estado anímico y de salud más aun de los niños, permite también la adquisición de nociones básicas las cuales influye en la identidad, nivel social, intelectual y emocional. Cabe mencionar que la acción de la psicomotricidad ejercicios físicos acelera las funciones vitales y mejoran el estado de ánimo proporcionando: salud y vitalidad, independencia, salud mental y socialización, es así como, los elementos fundamentales de la psicomotricidad son la: Motricidad fina, motricidad gruesa y esquema corporal. (Alberto, 2010)

La presente investigación se centra en analizar principalmente a la Motricidad Gruesa, considerado como el desarrollo motor grueso es el movimiento de los músculos de cuerpo de forma coordinada el cual mantiene el equilibrio, brinda agilidad 
y fuerza además esto se puede evidenciar desde los recién nacidos cuando empiezan a tener mejor posición de su cuerpo control de su cabeza, sedestación, giros, gatear hasta poco a poco continuar con el proceso de bipedestación hasta lograr ponerse de pie y mantenerse por sí solo. Es la capacidad que poseen los individuos para coordinar a partir de instrucciones del cerebro movimientos globales y amplios de su cuerpo, los que le permiten un equilibrio, el concepto motricidad tiene relación con la función de un ser vivo para generar movimientos por sí mismo, así sea de una sección del cuerpo o de su integridad siendo este un grupo de actos involuntarios coordinados y sincronizados por diferentes unidades motoras.

El niño y niña desarrolla habilidades motrices como: correr saltar. Lanzar, trepar, reptar, permitiéndoles fortalecer los músculos de su cuerpo y mantener el equilibrio a temprana edad. Para el área motora aplica dos leyes psicofisiológicas que permiten el movimiento, el céfalo caudal que es la consecución de movimientos desde la cabeza hacia los pies y el próximo distal que surge desde el eje central del cuerpo hacia las extremidades.

Esta habilidad es importante por que comienza a desarrollarse desde el vientre de la madre y cuando nacemos progresivamente la terminamos de desarrollar, esto hace que el ser humano pueda tener dominio de su propio cuerpo de tal forma que pueda reproducir movimientos gestos incluyendo la intuición, espontaneidad y coordinación así llegando alcanzar el desarrollo integral.

Es importante porque incluye movimientos musculares los cuales posibilitan al niño moverse, desplazarse los cuales deben ser controlados para fijar su precisión, además utiliza sus sentidos para procesar información del entorno que lo rodea contribuyendo a la parte cognitiva y del lenguaje.

Por tal motivo el desarrollo oportuno del área motora gruesa desde sus primeros años de vida marcara sus habilidades futuras, así es como Jean Piaget aporta con su teoría "la inteligencia se construye a partir de la actividad motriz del niño y niña", (Durivage, 1984, p.1).Lo que nos quiere decir con este aporte es que mientras el niño sea lo más pronto estimulado mayores conexiones neurológicas va a tener y mayor posibilidad de crear su propia información, alcanzando así su propia madurez psicomotriz.

El estimular el área motora gruesa permite al niño superar desafíos y reaccionar ante situaciones, como: Favorece el control y el dominio de su cuerpo, controla su equilibrio y respiración, maneja una buena postura, mejora su expresión corporal, coordina ritmo y tiempo, ayuda a la memoria, dominio de planos horizontal y vertical, se adapta mejor a su medio y facilita su orientación, domina nociones, organiza mejor su espacio, estimula la percepción de los objetos en relación a su cuerpo, apoya a la concentración y creatividad, estimula la expresión corporal, mejora la autoestima controla los impulsos y canaliza la energía y ayuda a enfrentar miedos e inseguridades.

Un ejemplo claro es cuando el niño se encuentra pateando pelota se puede pensar que solo está jugando, pero esta actividad va más allá de ser un simple juego a más de trabajar sus habilidades físicas su cerebro está activo recabando información mediante sus sentidos.

Las habilidades motrices son todas aquellas conductas y aprendizajes adquiridos por una persona, caracterizados por su espontaneidad y porque no responde a los modelos concretos de movimientos o gestos, esta forma es común de todos los individuos ya que han permitido la supervivencia del ser humano, está fundamentado en posteriores aprendizajes motrices. Las habilidades motoras básicas nos permiten 
desplazarnos, trabajar, jugar, relacionarnos

en

definitiva

vivir.

Estas habilidades dependen de la fuerza y resistencia de los músculos, las habilidades motoras gruesas comienzan a desarrollarse en la primera infancia desarrollándose primero en la parte alta del cuerpo y después en la parte baja.

De acuerdo con Godfrey y Kephart (1969), los movimientos fueron clasificados dos categorías básicas, como principal los movimientos que implican el manejo propio del cuerpo como es el caminar, correr, estar de pie o sentado, y secundario los movimientos en relación a los objetos en lo que corresponde a manipular, lanzar, golpear jugar etc., en cambio, otros autores engloban otras áreas concretas como el área Locomotrices, no locomotrices, proyección y percepción. (Rivero, 2010)

Existe las habilidades motrices específicas que se traducen a la relación de nuestro cuerpo con un objetivo de manera que cause precisión, por ejemplo, las habilidades de gimnasia, deportes lo cual causa beneficio en la formación corporal, buena postura, movimiento seguro y eficiencia motriz.

Según Fernando Sánchez en su libro Bases para una Didáctica de la Educación Física y el Deporte (1992), los niños desarrollan sus habilidades motrices y su evolución se da en 4 diferentes fases:

Primera Fase. - Corresponde a la edad de 4 a 6 años esta fase es receptiva, aquí es cuando comienza a entender la estructura y funcionamiento de su cuerpo en relación con el espacio que los rodea. Segunda fase. - entre 7 y 9 años aquí se desarrolla la motricidad básica sus movimientos son más complejos y progresivamente más perfectos. Tercera fase. -Inicia desde los 10 hasta los 13 años en esta fase el niño ya consolida todas sus habilidades y las relaciona a actividades deportivas y Cuarta fase. - corresponde a los 14 a 17 años aquí es donde el niño pule sus habilidades es capaz de inclinarse por deportes y otras disciplinas como una profesión o actividad extraescolar

Estas fases más las actividades motrices contribuyen a un desarrollo físico, cognitivo y emocional adecuado, por ello se debe trabajar en las destrezas motrices que es la capacidad de coordinar las extremidades con los diferentes segmentos del cuerpo y en las tareas motrices que es la ejecución de la destreza. El desarrollo de las habilidades motoras gruesas es necesario para el desarrollo de las habilidades motoras finas, para el desarrollo viso motor y perceptual.

Los elementos de la motricidad gruesa son: el dominio corporal estático, el dominio corporal dinámico y la coordinación general.

Dominio estático, son las actividades motrices que llevaran al niño o niña a interiorizar su esquema corporal en su totalidad o de forma segmentada, está conformada por elementos psicomotores los cuales son la Tonicidad muscular. Auto Control y la respiración:

Tonicidad muscular, Es un estado de ligera contracción en el cual los músculos se encuentran estirados su objetivo es servir como fondo para las actividades relacionadas con la postura y el movimiento intencional, es fuente de información receptiva (Berruezo, 2000).

Para desarrollar la tonicidad, las actividades se ofrecen en posiciones tales como: de pie, sentado, gateando y con movimientos y con diversosrados de dificultad

Autocontrol, Es la capacidad que tiene el niño para controlarse canalizando toda su energía en alguna actividad, por ello es importante el dominio de su tono muscular para tener un mejor control y postura adecuada. Se controla los músculos del cuello y su movimiento durante el primer mes y para el tercer y cuarto mes los músculos del tronco adquieren más firmeza logrando sujetar la cabeza, seguido de los brazos, para el octavo y noveno mes ya controla su cuerpo e inicia su proceso de descubrimiento 
al gatear o sostenerse de pie con ayuda, una vez dominado este proceso al año ya logra pararse solo incluso da sus primeros pasos.

Respiración, respirar es nuestro primer acto que realizamos al nacer, va más allá de nuestra de nuestra conciencia, es un proceso fisiológico que realizamos de manera independiente, cuando ya se separa del cordón umbilical a partir de ese momento es una actividad innata imprescindible para vivir.

El dominio dinámico,Consiste en la acción de grupos musculares en la ejecución de movimientos voluntarios complejos, es decir domina las extremidades superiores e inferiores para seguir con una consigna asignada permitiendo el desplazamiento y una adecuada sincronización de una manera armónica, indicando que sus músculos ya están madurando proporcionando confianza y seguridad en sí mismo ante cualquier situación.Existen ciertas áreas que facilitan el aprendizaje las cuales son: coordinación general, equilibrio, ritmo, coordinación viso motriz

La coordinación general, son los movimientos generales o globales de integración de las partes del cuerpo que conllevan a la armonía y desenvolvimiento del niño en sus diferentes etapas y edades, el desarrollo de la coordinación general influye en el proceso de lecto-escritura por lo que se refiere la parte física, psíquica y emocional de manera que no sea motivo de tensión y fluya una adecuada coordinación del cuerpo. Es necesario que el docente trabaje los contenidos desde la perspectiva motora así es como se puede reeducar los movimientos de caminar, escalar, subir, saltar gatear con la finalizar de optimizar esa energía y direccionarla al logro de aprendizajes, por ejemplo: El gateo,Trepar, Equilibrio, Ritmo, La Coordinación Viso motriz

Este, último se lleva a cabo mediante un estímulo visual interviene así la vista, el oído, el movimiento del cuerpo y el objeto con la capacidad del niño de responder ante ese estimulo e interpretarlo.

Implica movimientos controlados que requiere de atención y precisión en tareas donde implique el ojo y mano es conocido como coordinación viso-manual por ejemplo al realizar técnicas grafo plásticas. Ahora cuando un niño ejerce control de su cuerpo al patear una pelota, al desplazarse hacia un punto es una acción visomotriz en la cual responde músculos inferiores, al integrase con la coordinación visomanual logra un desempeño efectivo. "Se debe tener en cuenta que al dominar la coordinación viso motriz logrará un buen manejo manual”. (Rosas, 2013, p. 16)

El objetivo de esta investigación es buscar la relación de la expresión corporal en el desarrollo motor grueso de los niños de 3 años del Centro de Desarrollo Infantil "Orquídeas"

\section{METODOLOGÍA}

La investigación es de enfoque cualitativo, por que considera un abordaje de fenómenos sociales orientados a obtener información de conductas sobre el manejo del cuerpo mediante la expresión corporal. La metodología utilizada es la fenomenológica pues permite captar la experiencia y destrezas que y no presupone al mundo más allá de la experiencia, el nivel de la investigación es exploratorio descriptivo por que explora los contextos que muestra el objeto de estudio para la ejecución de la investigación en el ámbito de abordaje, en el espacio previsto, con las unidades de observación, se utilizó la estadística descriptiva para el análisis de la información, así se realizó la descripción de los resultados con su correspondiente análisis e interpretaciones cualitativas, tomando como base el marco teórico 
La modalidad de la investigación es de campo y bibliográfica, realizó en el sitio donde se presenta el problema, en el Centro de Desarrollo Infantil Orquídeas, donde se tomó contacto directo con la realidad y recabó información y datos de las diferentes manifestaciones e indicadores que presentan los niños de 3 años así como el bibliográfico - documental con el propósito de reforzar los resultados del análisis con el marco teórico, se obtuvieron datos e información de libros, manuales, revistas, e internet que constituyeron documentos de información primaria.

Para la presente investigación se solicitó el connsentimiento escrito del representante legal del Centro Infantil, coordinadora y padres de familia por motivo que es población infantil menor, de tal modo que sus datos serán utilizados únicamente con fines investigativos para buscar solución al problema planteado.

La técnica utilizada para la recolección de información fue la observación, con la aplicación de una matriz con destrezas relacionadas a las dos variables y considerando las establecidas en el currículo de educación inicial vigente, el instrumento utilizado fue una lista de cotejo en torno a destrezas y habilidades dependiente a su edad bajo el ámbito de expresión corporal y motricidad.

El proceso que se realizó para obtener los resultados estadísticos de niños fue la obtención de todas las frecuencias y porcentajes de cada uno de los ítems para finalmente contrastar los datos estadísticos con la parte teórica, obteniendo esos datos se relacionó lo practico con lo teórico y se procedió a la redacción.

\section{RESULTADOS}

La siguiente matriz recoge información de frecuencias con destrezas de acuerdo a lo recacabado por el investigador

Tabla 1:

Destrezas Relacionadas con la expresión corporal

\begin{tabular}{llccccccc}
\hline No. & \multicolumn{1}{c}{ Destrezas } & \multicolumn{2}{c}{ Iniciado } & \multicolumn{2}{c}{ Proceso } & \multicolumn{2}{c}{ Adquirido } \\
\hline $\mathbf{1}$ & \multicolumn{1}{c}{ Fre. } & $\begin{array}{l}\text { Porc. } \\
\text { Realizar movimientos articulatorios básicos: } \\
\text { sopla, intenta inflar globos, imita movimientos } \\
\text { de labios, lengua y mejillas. }\end{array}$ & 8 & 25 & 8 & 25 & 16 & 50 \\
\hline $\mathbf{2}$ & $\begin{array}{l}\text { Imita pasos de baile intentando reproducir los } \\
\text { movimientos y seguir el ritmo. }\end{array}$ & 10 & 31 & 10 & 31 & 12 & 38 \\
\hline $\mathbf{3}$ & $\begin{array}{l}\text { Realizar movimientos al escuchar canciones } \\
\text { intentando seguir el ritmo }\end{array}$ & 12 & 38 & 5 & 16 & 15 & 47 \\
\hline $\mathbf{4}$ & $\begin{array}{l}\text { Integrarse durante la ejecución de rondas bailes } \\
\text { y juegos tradicionales }\end{array}$ & 15 & 47 & 5 & 16 & 12 & 38 \\
\hline $\mathbf{5}$ & $\begin{array}{l}\text { Identificar algunas emociones y sentimientos de } \\
\text { las personas de su entorno y expresar las suyas } \\
\text { mediante el lenguaje no verbal. }\end{array}$ & 12 & 38 & 8 & 25 & 12 & 38 \\
\hline
\end{tabular}

En la destreza No.1, del $100 \%$ de niños observados en relación al indicador, realizar movimientos articulatorios básicos: sopla, intenta inflar globos, imita movimientos de labios, lengua y mejilla. El $50 \%$ se encuentra en adquirido, el $25 \%$ en proceso y el $25 \%$ iniciado. Los resultados obtenidos se dan a conocer que la mayoría de los niños logran cumplir con esta destreza y otros se encuentran en proceso, es importante saber que este tipo de movimientos con lengua y labios influyen de manera directa en el lenguaje verbal. 
En relación con la destreza No. 2, imita pasos de baile intentando reproducir los movimientos y seguir el ritmo. El $38 \%$ de niños a adquirido la destreza, mientras que el $31 \%$ se encuentra en proceso y el $31 \%$ está en iniciado, acorde a los resultados obtenidos indica que son pocos niños los que cumple con la destreza y que los demás niños se encuentran en proceso e iniciando su aprendizaje, es muy importante que la docente trabaje dominio dinámico en los niños a través de reconocer sonidos, intervalos y tiempos de manera que logre coordinar los movimientos.

Respecto de la destreza No. 3, realizar movimientos al escuchar canciones intentando seguir el ritmo. El $46 \%$ adquirió la destreza el $16 \%$ se encuentra en proceso y el $38 \%$ en iniciado, en esta pregunta se evidencia que son pocos los niños que está logrando el desarrollo de la destreza, se debe considerar que el ritmo es un elemento espontáneo del dominio corporal dinámico, el cual debe ser trabajado desde el estudio de una buena coordinación, tiempo y duración.

En relación con la destreza No. 4 sobre, integrarse durante la ejecución de rondas bailes y juegos tradicionales. El $37 \%$ se encuentra en adquirido, el $16 \%$ en proceso y el $47 \%$ en iniciado, se considera que los niños tienen problemas con el desarrollo de la confianza y seguridad ya que no han logrado integrarse en actividades artísticas que requieren de su expresión corporal y gestual.

En referencia a la destreza No. 5. identificar algunas emociones y sentimientos de las personas de su entorno y expresar las suyas mediante el lenguaje no verbal. El $38 \%$ ha adquirido el indicador, mientras que el $25 \%$ se encuentra en proceso y el $37 \%$ está en iniciando, frente a esta destreza existen pocos niños que han adquirido este aprendizaje, sin embargo, se debe enseñar a reconocer y gestionar las emociones logrando un favorable desarrollo de su autoestima y autoconcepto, lo cual permitirá una buena interrelación social y que gracias a su lenguaje corporal su mensaje exprese varias sensaciones al oyente.

Tabla 2:

Destrezas Relacionadas con la motricidad gruesa

No. Destrezas Iniciado Proceso Adquirido

\begin{tabular}{|c|c|c|c|c|c|c|c|}
\hline & & Fre. & Porc. & Fre. & Porc. & Fre. & Porc. \\
\hline 6 & $\begin{array}{l}\text { Realizar ejercicios de equilibrio dinámico } \\
\text { controlando los movimientos de las partes } \\
\text { gruesas del cuerpo. }\end{array}$ & 11 & 34 & 9 & 28 & 12 & 38 \\
\hline 7 & $\begin{array}{l}\text { Explorar diferentes formas de movimiento para } \\
\text { interiorizar su percepción global como parte de } \\
\text { su proceso de estructuración de la imagen } \\
\text { corporal }\end{array}$ & 12 & 38 & 8 & 25 & 12 & 38 \\
\hline 8 & $\begin{array}{l}\text { Caminar y correr coordinadamente manteniendo } \\
\text { el equilibrio a diferentes distancias orientaciones } \\
\text { y ritmos utilizando el espacio total }\end{array}$ & 8 & 25 & 12 & 38 & 12 & 38 \\
\hline 9 & $\begin{array}{l}\text { Mantener el control postural en diferentes } \\
\text { posiciones del cuerpo sentado, de pie, en } \\
\text { cuclillas, de cubito dorsal y cubito ventral }\end{array}$ & 7 & 22 & 14 & 44 & 11 & 34 \\
\hline 10 & $\begin{array}{l}\text { Realizar ejercicios que involucren movimientos } \\
\text { segmentados de partes gruesas del cuerpo, } \\
\text { cabeza, tronco y extremidades }\end{array}$ & 10 & 31 & 7 & 22 & 15 & 47 \\
\hline
\end{tabular}

En la destreza No. 6, sobre, realizar ejercicios de equilibrio dinámico controlando los movimientos de las partes gruesas del cuerpo. El $38 \%$ cumple con el indicador adquirido, mientras que el $28 \%$ se encuentra en proceso y el $34 \%$ está en iniciado, los resultados obtenidos son pocos los niños que han alcanzado la destreza, y los demás se encuentran en 
el proceso, lo que refleja que esta habilidad está siendo desarrollada al trabajar estabilidad, posición del cuerpo, tipos de desplazamientos con la finalidad de no caer y causarse daño.

En relación con la destreza No. 7, explorar diferentes formas de movimiento para interiorizar su percepción global como parte de su proceso de estructuración de la imagen corporal. El $38 \%$ se encuentra en adquirido, el $24 \%$ en proceso y el $37 \%$ en iniciado, os resultados obtenidos de la investigación dan cuenta que son pocos los niños que han logrado alcanzar la destreza, se conoce que para su edad el niño debe ser capaz de interpretar su cuerpo, sus posibilidades y limitaciones sobre su cuerpo y sus partes segmentadas.

En lo referente a la destreza No. 8, caminar y correr coordinadamente manteniendo el equilibrio a diferentes distancias orientaciones y ritmos utilizando el espacio total. El $38 \%$ cumple con el indicador mientras que el $24 \%$ se encuentra en proceso y el $38 \%$ está en iniciado, según los resultados obtenidos se da a conocer que son pocos los niños que dominan la destreza es importante desarrollar este tipo de actividades ya que generan confianza, independencia conjuntamente con el movimiento ayudando así a coordinar en diferentes tiempos e intervalos.

Del total de niños investigados en relación con la destreza No. 9, mantener el control postural en diferentes posiciones del cuerpo sentado, de pie, en cuclillas, de cubito dorsal y cubito ventral. El $34 \%$ cumple con el indicador mientras que el $44 \%$ se encuentra en proceso y el $22 \%$ está en iniciado. los datos expuestos de esta investigación indica que casi la mayoría de los niños se encuentra en proceso de adquirir esta destreza, teniendo en cuenta que es importante mantener una alineación correcta dentro del eje corporal con cada una de sus partes para lograr un trabajo coordinado y global.

Los niños observados en relación con la desteza No. 10, realizar ejercicios que involucren movimientos segmentados de partes gruesas del cuerpo, cabeza, tronco y extremidades. El $47 \%$ se encuentra en adquirido, el $22 \%$ en proceso y el $31 \%$ en iniciado, la mayor parte de niños han logrado desarrollar la destreza, gracias al desarrollo de la motricidad gruesa, el cuerpo del niño adquiere varias posibilidades de aprendizaje, además de la realización de diferentes movimientos articulados, coordinados y voluntarios de su cuerpo.

\section{DISCUSIÓN}

El propósito de la presente investigación fue describir la relación entre la expresión corporal y el desarrollo motor gruesa de niños de tres años del centro infantil Orquideas, a través del análisis de los resultados obtenidos en la observación realizada a este grupo de niños y contrastado teóricamente con artículos y documentos que den un aporte científico a la investigación.

El desarrollo de los niños por medio del desplazamiento se basa en las "diferentes actitudes innatas, adquiridas y expresivas de tipo motor que siente y comparte, al ingresar a la fase preescolar, el infante responderá a destrezas propias a su edad las cuales posibilitan conocer la capacidad de su cuerpo humano, su forma de tener relación y reaccionar frente a su entorno social. (Bolaños, 2006)

La Expresión Corporal entendemos el conjunto de técnicas corporales, espaciales y temporales que me permiten de forma artística expresar al otro los contenidos de mi mundo interior. Montesinos (2017)., esto lo logrará al desarrollar con movimientos articulatorios básicaos, cuando imite y reproduzca movimientos $Y$ sobre todo identifique emosiones es por ello por lo que tanto la expresión corporal como la motricidad gruesa estan íntimamente relacionados. 
En la destreza, identificar algunas emociones y sentimientos de las personas de su entorno y expresar las suyas mediante el lenguaje no verbal. Se obtuvo un resultado medianamente adquirido concluyendo que el área social incide también en la expresión corporal, ya que el infante aprende del vivir con los otros como podría ser positivamente alcanzando la felicidad o con ansiedad, temores, tensiones y aislamientos, por esto es fundamental la cooperación y colaboración igualitaria del conjunto, apreciando y estimulando su estabilidad y confianza. (Bolaños, 2006)

Por ello se considera que la educación preescolar debe ser un espacio que genere las primeras bases de confianza y esa libertad de crecer sin miedo, al compartir, cooperar jugar con otros niños, promoviendo así sus primeras experiencias emocionales.

Por mucho tiempo se ha desvalorizado la Educación Inicial sin dar importancia a el trabajo de los docentes al momento de realizar actividades recreativas que anteriormente se pensaba que era para perder el tiempo o que son juegos simples sin ningún objetivo, hoy en día el trabajo corporal no tiene límites, ya que es considerado como primer receptor de información y aprendizaje, así es como los niños desarrollan habilidades motoras, conceptos espaciales, vocabulario, percepciones es decir alcanza un desarrollo integral de sus capacidades.

Por ello los Centros Infantiles o instituciones de atención a niños en sus primeras edades están en el compromiso de potencializar cada una de sus capacidades, ya que medida que el niño va creciendo va desarrollando sin número de habilidades como es la consecución de dominios motrices, como es el dinámico y estático en actividades globales o segmentadas se asocia a la parte expresiva cuando se utiliza gestos, espacio compartidos, al trabajo con la utilización de elementos puestos en práctica en diferentes disciplinas como es el baile libre, danza, teatro desempañado un rol equilibrado, es decir se siente bien consigo mismo y lo expresa mediante su cuerpo. (Gonzalez, et al., 2000)

Nuestros resultados encuentran una gran diferencia entre el aporte de la institución con la intervención de las familias en sus hogares en lo que responde al rendimiento motor, varias familias limitan el aprendizaje de sus hijos, al no compartir con ellos tiempo y espacios recreativos, consideran que no deben ensuciarse negado su iniciativa por descubrimiento, Al respecto, Handal y Cols. afirman la relación padres e hijos favorece el desarrollo motor, cognitivo y social. (Handal, et al., 2007)

La información arroja también que el desarrollo motor está ligado a movimientos más complejos y precisos bajo el dominio del sistema nervioso, el cual es el encargado de controlar y coordinar la actividad muscular. (Cabezuelo, et al., 2016), de tal modo que el docente debe conocer cada una de las etapas de desarrollo psicomotor para comprender que necesita desarrollar para su edad el niño,

Los datos encontrados también indican que existe un escaso material, espacio y desconocimiento por parte del personal al mantener la misma didáctica de años atrás con los niños. En resumen, este estudio destaca de manera particular al desarrollo motor y la expresión corporal como habilidades que van de la mano que se ven desarrolladas cuando se trabaja una buena estimulación en el hogar e instituciones, sin embargo, cabe destacar la libertad del niño al explorar su medio sin forzar sus acciones aceptando su naturalidad su presencia y su conducta.

Finalmente, los resultados encontrados dejan ver relacion que existe entre la expresión corporal y el desarrollo motor grueso, eso implica que mientras mayor capacidad de lenguaje corporal, memoria y el esquema corporal sea desarrollado en el niño logrará un dominio en la coordinación de movimientos amplios de la 
locomoción, el niño desarrolla habilidades motrices como: correr saltar. Lanzar, trepar, reptar, permitiéndoles fortalecer los músculos de su cuerpo y mantener el equilibrio a temprana edad. (Alberto, 2010). Por ello la necesidad de establecer programas de educación a padres y docentes que fomenten una estimulación adecuada y compromiso por promover el desarrollo integral media la expresión corporal para el desarrollo motor grueso.

\section{REFERENCIAS}

Antolín Jimeno, L. (2013). Expresión corporal: Fundamentos motrices. Publicaciones de la Universidad de Valencia (PUV). https://www.digitaliapublishing.com/a/34933

Alexis, J. H., Betsy, L., Breilh, J., \& Siobán, D. H. (mayo de 2007). Sociodemographic and nutritional correlates of neurobehavioral development: a study of young children in a rural region of Ecuador. Pan American Journal of Public Health, 21(5), 292-300. Obtenido de http://www.scielosp.org/scielo.php?script=sci_arttext\&pid=S102049892007000400004

Berruezo, P. P. (2003). Psicomotric idad en el ámbito educativo. Obtenido de https://www.um.es/cursos/promoedu/psicomotricidad/2005/material/contenido s-psicomotricidad-texto.pdf

Blanco Vega, M. d. (Septiembre de 2009). Enfoques teóricos sobre la expresión corporal como medio de formación y comunicación. Corporación Universitaria Iberoamericana, 11(1), 15-28. doi:ISSN-e 0123-8264 obtenido de https://horizontespedagogicos.ibero.edu.co/article/view/332/298

Bolaños Bolaños, G. (2006). Educación por medio del movimento y expresión corporal (Primera ed.). Costarica: Universidad estatal. doi:ISBN: 9977-64-277-X

Cabezuelo Huerta, G., \& Frontera Izquierdo, P. (2016). El desarrollo Psicomotor. Marcea. doi:ISBN: 978-84-277-1846-3

Consejo Nacional de Planificacion. (13 de Julio de 2015). Plan Nacional del Buen Vivir 2013-2017. Obtenido de http://www.cpccs.gob.ec/wpcontent/uploads/2015/12/PNBV-2013-2017.pdf

Educación, M. d. (2017). Curriculo de Educación Inicial. Obtenido de https://web.oas.org/childhood/ES/Lists/Recursos\%20\%20Planes\%20Nacional es/Attachments/395/9.\%20Curriculo\%20Institucional\%20de\%20Educaci\%C3 $\%$ B3n.pdf

Gonzalez, M., Hernández, M. D., Martinez, A., \& Ureña , F. (2000). La Educación Física en Secundaria. Fundamentación teórica (Segunda ed.). INDE. doi:9788487330674

Lení Nista-Piccolo, V., \& Wey Moreira, W. (2015). Movimiento y expresión corporal: En Educación Infantil. Narcea Ediciones. https://www.digitaliapublishing.com/a/67325

MIES. (2014). Norma Técnica . Obtenido de https://www.inclusion.gob.ec/wpcontent/uploads/downloads/2014/03/Vista-Previa-Norma-Te\%CC\%81cnicaCIBV-15x21-32-pag-Final-05-03-142.pdf

Ministerio de Educación. (2014). Curriculo de Educación Inicial. Obtenido de https://educacion.gob.ec/wp-content/uploads/downloads/2014/06/curriculoeducacion-inicial-lowres.pdf

Montesinos Ayala, D. (2017). La expresión corporal: Su enseñanza por el método natural evolutivo. Inde. https://www.digitaliapublishing.com/a/103578 
Rodriguez Vaquero, V. (2008). El mejoramiento de la salud a traves de la expresión corporal. Reflexiones, 127-137. Obtenido de https://www.redalyc.org/articulo.oa?id=72912553009

Rogoff. (1993). Terras.edu.ar. Obtenido de http://www.terras.edu.ar/biblioteca/6/PE_Rogoff_Unidad_2.pdf

Rosas, R. (18 de 7 de 2013). http://www.dspace.uce.edu.ec/. Obtenido de http://www.dspace.uce.edu.ec/bitstream/25000/3427/1/T-UCE-0010-458.pdf

Toro Santacruz , Soraya; Espinoza Salas, Maria. (2014). Curriculo de Educación Inicial. Obtenido de https://educacion.gob.ec/wpcontent/uploads/downloads/2014/06/curriculo-educacion-inicial-lowres.pdf

Uribe, A. (10 de Octubre de 2017). AlTTA. Obtenido de Psicologia infantil y juvenil: http://www.aitta.es/index.php/la-memoria-corporal/

Villacres, A. P. (2014). Curriculo de Educacion Iniciai. Obtenido de http://parvulariasdelecuador.blogspot.com/2014/06/curriculo-educacioninicial.html 